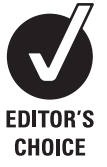

${ }^{1}$ Department of Cardiology, Leiden University Medical Centre, Leiden, The Netherlands;

${ }^{2}$ The Interuniversity Cardiology Institute of the Netherlands, Utrecht, The Netherlands; ${ }^{3}$ Department of Cardiology, Kaunas University of Medicine, Kaunas, Lithuania; ${ }^{4}$ Department of Radiology, Leiden University Medical Centre, Leiden, The Netherlands

Correspondence to:

Dr J J Bax, Department of Cardiology, Leiden University Medical Centre, Postal zone: C5-P, P0 Box 9600, 2333 ZA Leiden, The Netherlands: j.j.bax@lumc.nl

Accepted 8 September 2009

\title{
Plaque type and composition as evaluated non- invasively by MSCT angiography and invasively by VH IVUS in relation to the degree of stenosis
}

\author{
J E van Velzen, ${ }^{1,2}$ J D Schuijf, ${ }^{1}$ F R de Graaf, ${ }^{1}$ G Nucifora, ${ }^{1}$ G Pundziute, ${ }^{1,3}$ \\ J W Jukema, ${ }^{1,2}$ M J Schalij, ${ }^{1}$ L J Kroft, ${ }^{4}$ A de Roos, ${ }^{4}$ J H C Reiber, ${ }^{4}$ E E van der Wall, ${ }^{1,2}$ \\ J J $\operatorname{Bax}^{1}$
}

\begin{abstract}
Background: Imaging of coronary plaques has traditionally focused on evaluating degree of stenosis, as the risk for adverse cardiac events increases with stenosis severity. However, the relation between plaque composition and severity of stenosis remains largely unknown. Objective: To assess plaque composition (non-invasively by multislice computed tomography (MSCT) angiography and invasively by virtual histology intravascular ultrasound (VH IVUS)) in relation to degree of stenosis.
\end{abstract}

Methods: 78 patients underwent MSCT (identifying three plaque types; non-calcified, calcified, mixed) followed by invasive coronary angiography and VH IVUS. VH IVUS evaluated plaque burden, minimal lumen area and plaque composition (fibrotic, fibro-fatty, necrotic core, dense calcium) and plaques were classified as fibrocalcific, fibroatheroma, thin-capped fibroatheroma (TCFA), pathological intimal thickening. For each plaque, percentage stenosis was evaluated by quantitative coronary angiography. Significant stenosis was defined $>50 \%$ stenosis. Results: Overall, 43 plaques (19\%) corresponded to significant stenosis. Of the 227 plaques analysed, 70 were non-calcified plaques (31\%), 96 mixed (42\%) and 61 calcified (27\%) on MSCT. Plaque types on MSCT were equally distributed among significant and non-significant stenoses. VH IVUS identified that plaques with significant stenosis had higher plaque burden (67\% (11\%) vs $53 \%$ $(12 \%), p<0.05)$ and smaller minimal lumen area $(4.6$ (3.8-6.8) mm² vs $\left.7.3(5.4-10.5) \mathrm{mm}^{2}, \mathrm{p}<0.05\right)$. Interestingly, no differences were observed in percentage fibrotic, fibro-fatty, necrotic core and dense calcium. Nonsignificant stenoses were more frequently classified as pathological intimal thickening $(46(25 \%)$ vs $3(7 \%)$, $p<0.05$ ), although TCFA (more vulnerable plaque) was distributed equally $(p=0.18)$.

Conclusion: No evident association exists between the degree of stenosis and plaque composition or vulnerability, as evaluated non-invasively by MSCT and invasively by VH IVUS.

Imaging of atherosclerosis has traditionally focused on evaluating the degree of stenosis, as the risk for adverse cardiac events increases with stenosis severity. ${ }^{1}$ However, plaque composition rather than degree of stenosis may have a pivotal role in the development of acute atherothrombotic events and sudden cardiac death. Indeed, rupture of a vulnerable plaque (plaque with a large necrotic core, macrophage infiltration and thin fibrous cap) has been considered to be the primary cause of acute coronary syndromes (ACS). ${ }^{2}$ In addition, it has been shown that almost two-thirds of vulnerable plaques are located in non-obstructive atherosclerotic lesions. ${ }^{34}$ Moreover, non-obstructive lesions outnumber the more severely obstructive lesions and therefore account for the majority of ruptured plaques. ${ }^{5}{ }^{6}$ Possibly, direct visualisation of atherosclerosis rather than merely identifying obstructive disease may improve risk stratification for future events.

Multislice computed tomography (MSCT) has been demonstrated to be a promising tool for noninvasive assessment of atherosclerotic plaque burden and composition. ${ }^{7}$ An important advantage of MSCT is that the technique not only visualises luminal narrowing but can also identify atherosclerotic plaque in the arterial vessel wall. ${ }^{78}$ Accordingly, in contrast to invasive coronary angiography, lesions that show outward (positive) remodelling without luminal narrowing can also be easily identified. In addition, information on plaque composition can be obtained and lesions can be differentiated into non-calcified, mixed or calcified. Interestingly, retrospective studies comparing plaque composition on MSCT between patients presenting with ACS and stable coronary artery disease (CAD) showed that non-calcified and mixed plaques were associated with ACS. ${ }^{9} 10$

With respect to invasive techniques, virtual histology intravascular ultrasound (VH IVUS) is a promising modality for the assessment of coronary plaque characteristics in vivo. VH IVUS has been validated against histopathology and shows good accuracy for the determination of different plaque components such as fibrotic, fibro-fatty, necrotic core and dense calcium. ${ }^{11}$ The predictive accuracy of VH IVUS plaque composition assessment, when compared to histopathology, was $90.4 \%$ for fibrous, $92.8 \%$ for fibro-fatty, $89.5 \%$ for necrotic core and $90.9 \%$ for dense calcium regions. Using this technique, it was demonstrated that differences in plaque composition can be related to particular clinical settings. Indeed, in a study by Rodriguez-Granillo et al, VH IVUS-derived thincapped fibroatheroma (vulnerable plaque) was found more often in patients with ACS than in patients with stable CAD. ${ }^{12}$ Accordingly, VH IVUS may be useful for the evaluation of atherosclerotic plaque composition in vivo.

The purpose of this study was to evaluate if differences in plaque composition are related to the degree of stenosis. To this end, plaque composition 
was evaluated non-invasively by MSCT, followed by invasive evaluation using VH IVUS.

\section{METHODS}

\section{Patients and study protocol}

The study group consisted of 78 symptomatic patients who presented at the outpatient clinic (Leiden, The Netherlands) for the evaluation of chest pain and underwent MSCT coronary angiography, followed within a month by clinically referred invasive coronary angiography and VH IVUS of one to three vessels. Contraindications for MSCT were (1) (supra) ventricular arrhythmias, (2) renal insufficiency (glomerular filtration rate $<30 \mathrm{ml} / \mathrm{min}$ ), (3) known allergy to iodine contrast material, (4) severe claustrophobia, (5) pregnancy. Exclusion criteria for IVUS were severe vessel tortuosity, severe stenosis or vessel occlusion. In each patient, the presence of CAD risk factors such as diabetes mellitus, systemic hypertension, hypercholesterolaemia, positive family history, smoking and obesity, were recorded. The Framingham 10-year risk for hard CAD events was calculated as previously described in the National Cholesterol Education Program's Adult Treatment Panel III report. ${ }^{13}$ Subsequently, the study population was then categorised as at low $(<10 \%)$, intermediate (10-20\%) and high risk (>20\%).

\section{MSCT}

\section{Data acquisition}

MSCT coronary angiography was performed using either a 64detector row helical scanner (Aquilion 64, Toshiba Multi-slice system, Toshiba Medical Systems, Otawara, Japan) or a 320detector row volumetric scanner (Aquilion ONE, Toshiba Medical Systems, Otawara, Japan). Unless contraindicated, if the heart rate was $\geqslant 65$ beats/min, $\beta$-blockers (metoprolol $50 \mathrm{mg}$ or $100 \mathrm{mg}$, single dose, 1 hour before examination) were administered. For the 64-slice contrast-enhanced scan, collimation was $64 \times 0.5 \mathrm{~mm}$, tube voltage $100-135 \mathrm{kV}$ and tube current 250-350 mA, depending on body shape. Non-ionic contrast material (Iomeron 400, Bracco, Milan, Italy) was administered with an amount of $80-110 \mathrm{ml}$ followed by a saline flush with a flow rate of $5 \mathrm{ml} / \mathrm{s}$. For the 320 -slice contrastenhanced scan the heart was imaged in a single heartbeat, using prospective triggering with exposure interval depending on the heart rate. Scan parameters were $350 \mathrm{~ms}$ gantry rotation time, $100-135 \mathrm{kV}$ tube voltage and a tube current of 400-580 mA, depending on body mass index. In total, $60-90 \mathrm{ml}$ contrast material (Iomeron 400) was administered with a rate of 5-6 ml/s followed by a saline flush. Subsequently, datasets were reconstructed and transferred to a remote workstation as previously described. ${ }^{14}$

\section{Coronary plaque assessment}

MSCT angiograms were evaluated using dedicated software (Vitrea 2.0 or Vitrea FX 1.0, Vital images, Minnetonka, MN, USA). MSCT angiographic examinations were evaluated in consensus by two experienced readers including an interventional cardiologist blinded to conventional coronary angiography and IVUS findings. According to the modified American Heart Association classification, coronary arteries were divided into 17 segments. ${ }^{15}$ Each segment was evaluated for the presence of any atherosclerotic plaque using axial and/or orthogonal images and curved multiplanar reconstructions. Structures $>1 \mathrm{~mm}^{2}$ within and/or adjacent to the coronary artery lumen, which could be clearly distinguished from the vessel lumen, were defined as plaques. ${ }^{7}$ By segment, one coronary plaque was selected at the site of the most severe luminal narrowing. To verify the presence of calcifications, the calcium score was evaluated before determining plaque composition. Plaques were further classified as: (1) non-calcified plaque (plaques with lower CT (computed tomography) attenuation compared to contrast-enhanced lumen without any calcification), (2) mixed plaque (non-calcified and calcified elements in single plaque), (3) calcified plaque (plaques with high CT attenuation compared to contrast-enhanced lumen).

\section{Conventional coronary angiography}

Conventional coronary angiography was performed according to standard protocols. Vascular access was obtained through the femoral approach according to the Seldinger technique with the use of a $6 \mathrm{~F}$ or $7 \mathrm{~F}$ sheath. Quantitative coronary angiography (QCA) analysis was performed by an observer unaware of MSCT and IVUS findings with the use of OCA-CMS version 6.0 (Medis, Leiden, The Netherlands). For each plaque examined both with MSCT and VH IVUS, the percentage diameter stenosis as measured by quantitative coronary angiography was reported. Measurements were performed on at least two orthogonal projections. The highest percentage diameter stenosis was used for further analysis. Significant stenosis was defined as $>50 \%$ stenosis.

\section{VH IVUS}

\section{Image acquisition}

VH IVUS examinations were acquired during coronary angiography in 136 of the 225 available vessels with the use of a dedicated IVUS-console (Volcano Corporation, Rancho Cordova, CA, USA). After administration of nitrates locally, VH IVUS was performed with a $20 \mathrm{MHz}, 2.9 \mathrm{~F}$ phased-array IVUS catheter (Eagle Eye, Volcano Corporation, Rancho Cordova, CA, USA), which was introduced distally in the coronary artery. A speed of $0.5 \mathrm{~mm} / \mathrm{s}$ was used for motorised automated pullback until the catheter reached the guiding catheter. Images were stored on CDRom or DVD for offline analysis.

\section{Image analysis}

VH IVUS analysis was performed by two experienced observers blinded to baseline patient characteristics with the use of dedicated software (pcVH 2.1, Volcano Corporation, Rancho Cordova, CA, USA). The lumen and the media-adventitia interface were defined by automatic contour detection and on all individual frames manual editing was performed. All four plaque components were differentiated into different colour codes (fibrotic tissue being labelled in dark green, fibro-fatty in light green, necrotic core in red and dense calcium in white), as validated previously. ${ }^{16}$ For each frame, vessel and lumen area were calculated and percentage plaque burden was calculated as plaque plus media cross-sectional area (CSA) divided by external elastic membrane CSA multiplied by $100 .{ }^{17}$ Plaque composition was calculated as percentage of the plaque burden.

Qualitative evaluation of plaque type was visually assessed at the minimal lumen area site on IVUS using the following classification:

(i) Pathological intimal thickening (PIT); defined as a mixture of fibrous and fibro-fatty tissues, a plaque burden $\geqslant 40 \%$ and $<10 \%$ necrotic core and dense calcium.

(ii) Fibroatheroma (FA); defined as having a plaque burden $\geqslant 40 \%$ and a confluent necrotic core occupying $10 \%$ of the plaque area or greater in three successive frames with evidence of an overlying fibrous cap. 
Figure 1 An example of combination of non-invasive imaging with 320-slice multislice computed tomography (MSCT) angiography and invasive imaging with coronary angiography and virtual histology intravascular ultrasound (VH IVUS). A 53-year-old woman was referred because of chest pain and intermediate risk profile, exercise testing was inconclusive and the patient was referred for anatomical evaluation by MSCT. An intermediate lesion in the mid left anterior descending coronary artery (LAD) was identified on MSCT. Consequently, the patient was further referred for coronary angiography combined with (VH) IVUS.

(A) Three-dimensional reconstruction depicting a lesion (arrow) with intermediate luminal narrowing in the mid LAD. (B) Curved multiplanar reconstruction of the $L A D$ and the corresponding lesion (arrow) showing a mixed plaque. (C) The findings were confirmed on conventional coronary angiography and quantitative coronary angiography (OCA) analysis

demonstrating luminal narrowing of $42 \%$ (arrow). (D) Corresponding VH IVUS image showing a substantial amount of necrotic core (labelled in red).
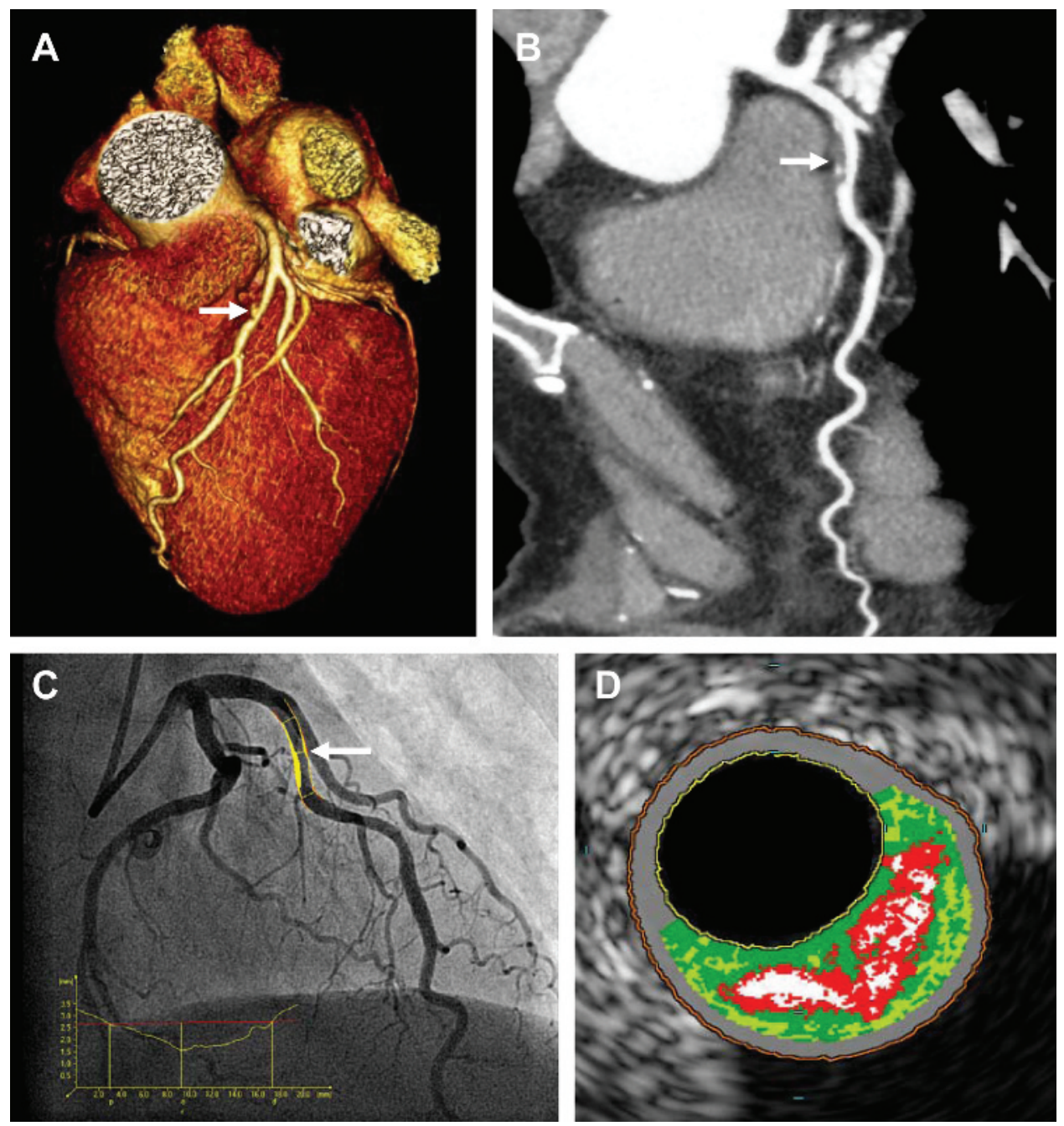

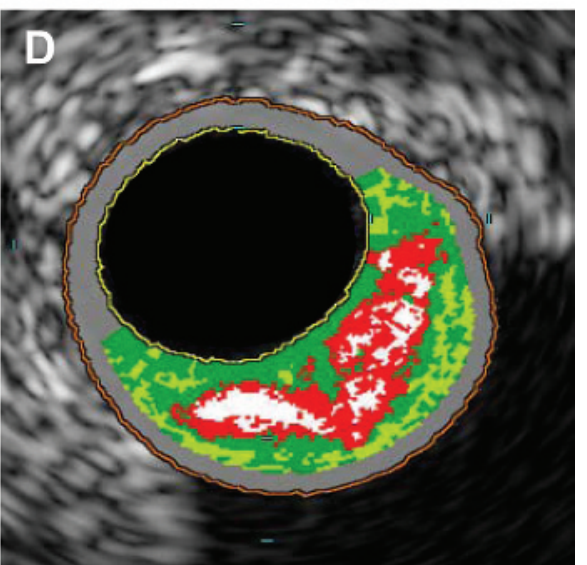

(iii) Thin-capped fibroatheroma (TCFA); defined as a lesion with a plaque burden $\geqslant 40 \%$, the presence of confluent necrotic core of $>10 \%$, and no evidence of an overlying fibrous cap.

(iv) Fibrocalcific plaque (FC); defined as a lesion with a plaque burden $\geqslant 40 \%$, being mainly composed of fibrotic tissue, having dense calcium $>10 \%$ and a confluent necrotic core of $<10 \%$ (higher amount accepted if necrotic core was located exclusively behind the accumulation of calcium). ${ }^{18} 19$

To match plaques identified on MSCT with plaques identified on VH IVUS; landmarks such as coronary ostia, side-branches and calcium deposits were used. Distances from the landmarks to the lesion were measured on multiplanar reconstructions on MSCT and matched with the longitudinal images of VH IVUS.

\section{Statistical analysis}

Continuous values are expressed as means (SD) if normally distributed and compared with the two-tailed $t$ test for independent samples. If not normally distributed, values are expressed as medians (interquartile range) and compared with the two-tailed Mann-Whitney test. Categorical values are expressed as number (\%) and compared between groups with two-tailed $\chi^{2}$ test. Binary logistic regression analysis was used to calculate the association of significant stenosis with the different plaque types as identified on MSCT or VH IVUS. A $\mathrm{p}$ value of $<0.05$ was considered statistically significant. Statistical analysis was performed using SPSS 14.0 software.

\section{RESULTS}

\section{Patients and study protocol}

In total, 78 patients were identified that had undergone MSCT and invasive coronary angiography with VH IVUS. A patient example is provided in figure 1 . Three patients were excluded owing to the absence of any identifiable plaques on MSCT. In all patients MSCT angiograms and VH IVUS studies were of diagnostic image quality. Characteristics of the remaining 75 patients are summarised in table 1 . In 89 vessels (40\%) VH IVUS could not be performed owing to severe vessel stenosis, vessel tortuosity, vessel occlusion or time limitations in the catheterisation laboratory. As a result, 136 vessels (60\%) were available for analysis. In total, 227 plaques were identified on MSCT in which corresponding VH IVUS analysis was available. On QCA, the average percentage stenosis of the plaques was $29 \%(9 \%)$. Forty-three plaques (19\%) corresponded to a significant stenosis (average percentage stenosis 61\% (9\%)) and 184 plaques (81\%) corresponded to a non-significant stenosis (average percentage stenosis $22 \%(2 \%))$. 
Table 1 Patient characteristics of study population

\begin{tabular}{ll}
\hline & No (\%) \\
\hline Gender (M/F) & $43 / 32$ \\
Age (years) & $59(11)$ \\
Risk factors for CAD & \\
$\quad$ Diabetes mellitus & $22(29 \%)$ \\
Hypertension & $45(60 \%)$ \\
$\quad$ Hypercholesterolaemia & $57(76 \%)$ \\
Positive family history & $33(44 \%)$ \\
Current smoking & $38(51 \%)$ \\
Obese (BMI $\left.\geqslant 30 \mathrm{~kg} / \mathrm{m}^{2}\right)$ & $14(19 \%)$ \\
Previous CAD & \\
$\quad$ Previous myocardial infarction & $20(27 \%)$ \\
Framingham risk score & \\
$\quad$ Low & $45(60 \%)$ \\
Intermediate & $22(29 \%)$ \\
$\quad$ High & $8(11 \%)$ \\
Heart rate (bpm) during MSCT & $62(9)$ \\
Prevalence of significant CAD (defined as at least 1 stenosis & $29(39 \%)$ \\
with $>50 \%$ luminal narrowing on OCA) & \\
\hline
\end{tabular}

CAD, coronary artery disease; BMI, body mass index; MSCT, multislice computed tomography; $\mathrm{OCA}$, quantitative coronary angiography.

\section{Compositional characteristics in relation to angiographic degree of stenosis}

Composition on MSCT

Of the 227 plaques analysed, 70 were non-calcified plaques $(31 \%), 96$ were mixed $(42 \%)$ and 61 were calcified $(27 \%)$ on MSCT. The different plaque types as identified by MSCT were equally distributed among significant and non-significant stenoses (fig 2). Plaque type on MSCT was not significantly related to stenosis (non-calcified plaque; OR 0.9 (95\% CI 0.4 to 1.8 ), $p=0.68$, mixed plaque; $O R 1.5$ (0.8 to 1.9$), p=0.26$ ); calcified plaque OR 0.7 (0.3 to 1.7$), \mathrm{p}=0.41$ ).

\section{Composition on VH IVUS}

In 136 vessels and 227 plaques, VH IVUS was successfully performed. The average plaque length analysed was $22(17) \mathrm{mm}$. Overall, the most prevalent plaque component was fibrotic tissue $(52 \%(12 \%))$, followed by fibro-fatty tissue $(22 \%(16 \%))$, necrotic core $(15 \%(9 \%))$ and dense calcium $(11 \%(10 \%))$. As expected and can be derived from table 2, plaques with a significant stenosis had a significantly higher plaque burden $(67 \%(11 \%)$ versus $53 \%(12 \%), \mathrm{p}<0.05)$ and smaller minimum lumen area $\left(4.6(3.8-6.8) \mathrm{mm}^{2}\right.$ versus $7.3(5.4-10.5) \mathrm{mm}^{2}$, $\mathrm{p}<0.05)$ compared to plaques without significant stenosis. Interestingly, no differences were observed in the amount of fibrotic tissue $(51 \%(10 \%)$ versus $53 \%(13 \%), p=0.62)$, fibrofatty tissue $(21 \%(9-33 \%)$ versus $18 \%(10-29 \%), p=0.42)$, necrotic core $(15 \%(8 \%)$ versus $15 \%(9 \%), \mathrm{p}=0.95)$ and dense calcium (8\% (3-15\%) versus $9 \%(3-15 \%), p=0.77)$.

Qualitative visual evaluation of coronary plaques revealed that pathological intimal thickening was more frequently observed in lesions with non-significant stenosis $(3(7 \%)$ versus $46(25 \%), \mathrm{p}<0.05)$; however, the more vulnerable plaques (thincapped fibroatheroma) were distributed equally (10 (23\%) versus $27(15 \%), p=0.18)$ (fig 3$)$. Pathological intimal thickening was significantly related to non-significant stenosis ( $\mathrm{OR}$ for significant stenosis 0.2 ( $95 \%$ CI 0.1 to 0.8$), p=0.02$ ), although there was no relation between the more vulnerable plaque types (thin-capped fibroatheroma) and significant stenosis (OR 1.8 (0.8 to 4.0$), p=0.18)$.

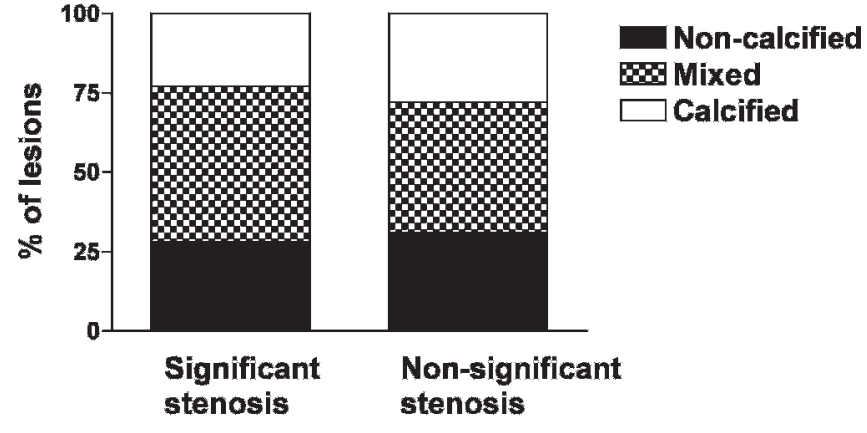

Figure 2 Bar graph demonstrating the relative distribution of different plaque types as determined by multislice computed tomography (MSCT) in lesions with significant and non-significant stenosis $(p=n s)$.

\section{DISCUSSION}

In the present study differences in plaque composition and vulnerability between lesions with significant and non-significant stenosis were assessed using non-invasive MSCT angiography and invasive VH IVUS. The main findings of coronary plaque characterisation using MSCT and VH IVUS can be summarised as follows.

No differences in plaque composition between significant and non-significant stenosis were demonstrated non-invasively by MSCT and invasively by VH IVUS. MSCT showed that the proportion of non-calcified, mixed and calcified plaques were similar between significant and non-significant lesions. VH IVUS confirmed these findings, revealing no differences in plaque composition (fibrotic, fibro-fatty, necrotic core and dense calcium) between significant and non-significant stenosis. Importantly, no differences were observed in plaque vulnerability between significant and non-significant stenosis as demonstrated by an even distribution of thin-capped fibroatheroma and percentage necrotic core.

In the present study non-calcified, mixed and calcified plaques on MSCT were equally distributed between lesions with significant and non-significant stenosis. The observations on MSCT corresponded to the findings on VH IVUS, indicating that plaque composition did not differ between lesions with significant and non-significant stenosis. These results are in line with previous studies exploring plaque composition with grey scale IVUS in relation to luminal narrowing on coronary angiography. ${ }^{20}$

Nonetheless, qualitative evaluation of plaque type on $\mathrm{VH}$ IVUS revealed more early stage atherosclerosis in non-significant stenosis, as reflected by a higher prevalence of pathological intimal thickening. On the other hand, one would also expect to find more advanced plaque composition in lesions with significant stenosis, including a higher degree of calcium. Indeed, other non-invasive imaging techniques, such as calcium scoring on electron beam computed tomography, have demonstrated that calcium is more prevalent in patients with larger atherosclerotic plaque burden and that a moderate relation exists between the extent of coronary calcium and presence of obstructive CAD. ${ }^{21}$ However, in the present study, no differences were found between the prevalence of calcified plaques on MSCT in significant and non-significant stenosis. These findings were confirmed by an even distribution of dense calcium on VH IVUS. This could possibly be explained by the fact that there is a marked discrepancy between apparent angiographic luminal narrowing and actual extent of atherosclerosis. Indeed, owing to compensatory enlargement or 
Table 2 Differences in plaque composition between lesions with significant and non-significant stenosis

\begin{tabular}{lccc}
\hline & $\begin{array}{l}\text { Significant stenosis } \\
(\mathbf{n}=\mathbf{4 3})\end{array}$ & $\begin{array}{l}\text { Non-significant stenosis } \\
(\mathbf{n}=\mathbf{1 8 4})\end{array}$ & p Value \\
\hline Plaque burden (\%) & $67(11)$ & $53(12)$ & $<0.05$ \\
Minimal lumen area $\left(\mathrm{mm}^{2}\right)$ & $4.6(3.8-6.8)$ & $7.3(5.4-10.5)$ & $<0.05$ \\
\% Fibrotic & $51(10)$ & $53(13)$ & 0.62 \\
\% Fibro-fatty & $21(9-33)$ & $18(10-29)$ & 0.42 \\
\% Necrotic core & $15(8)$ & $15(9)$ & 0.95 \\
\% Dense calcium & $8(3-15)$ & $9(3-15)$ & 0.77 \\
\hline
\end{tabular}

positive remodelling, a substantial build-up of plaque can occur before resulting in luminal narrowing and reduction of blood flow. Therefore, plaque composition gradually becomes more advanced while remaining non-significant (in terms of luminal narrowing) for a considerable period of time. Of note, recently Sipahi and co-workers demonstrated that type of arterial remodelling (negative or positive) does not predict subsequent progression of atherosclerosis in patients undergoing statin therapy. ${ }^{22}$ Interestingly, similar observations to our results were reported by Mintz et al using IVUS. The authors reported that coronary calcium correlated well with plaque burden but not with angiographical luminal narrowing. ${ }^{23}$ Accordingly, angiographic intermediate lesions were as likely to contain significant amounts of calcium as severe lesions.

Interestingly, there was no difference in the proportion of non-calcified and mixed lesions observed on MSCT among lesions with significant and non-significant stenosis. Although data are scarce, it has been suggested that non-calcified and mixed plaques on MSCT may be associated with increased plaque vulnerability. ${ }^{10} 24$ Indeed, studies comparing plaque patterns on MSCT between patients presenting with ACS and stable CAD have consistently shown that non-calcified and mixed plaques are more prevalent in patients with ACS. ${ }^{925}$ In addition, these lesions have been demonstrated to correspond with a larger amount of necrotic core and a higher prevalence of thin-capped fibroatheroma on VH IVUS. ${ }^{10}$ Accordingly, these observations suggest that the observation of non-calcified or mixed plaque on MSCT may potentially be of clinical relevance, regardless of stenosis severity.

Importantly, the observation of similar plaque composition between significant and non-significant stenosis translated into no differences in plaque vulnerability on VH IVUS, demonstrated by an even distribution of thin-capped fibroatheroma and percentage necrotic core on VH IVUS. Similar observations have been reported by serial angiographic studies showing that vulnerability was poorly related to angiographic degree of luminal narrowing. ${ }^{4}{ }^{26}$ In line with these studies, investigations directly addressing plaque composition also reported a lack of agreement between plaque vulnerability and degree of stenosis. Using conventional grey scale IVUS, Yamagishi et al prospectively examined coronary plaques with IVUS and found no differences in degree of luminal narrowing between plaques before ACS and plaques that remained stable. ${ }^{27}$ In addition, Saam et al studied carotid arteries of 192 subjects with non-invasive
Figure 3 Differences in percentage of visually assessed plaque types on virtual histology intravascular ultrasound (VH IVUS) between lesions with significant and non-significant stenosis. Significantly more lesions classified as pathological intimal thickening (PIT) were present in non-significant stenosis compared to significant stenosis (A). Percentage of fibroatheroma (FA), thin-capped fibroatheroma (TCFA) and fibrocalcific (FC) plaques were not significantly different between lesions with significant and non-significant stenosis (C, B, D).
A

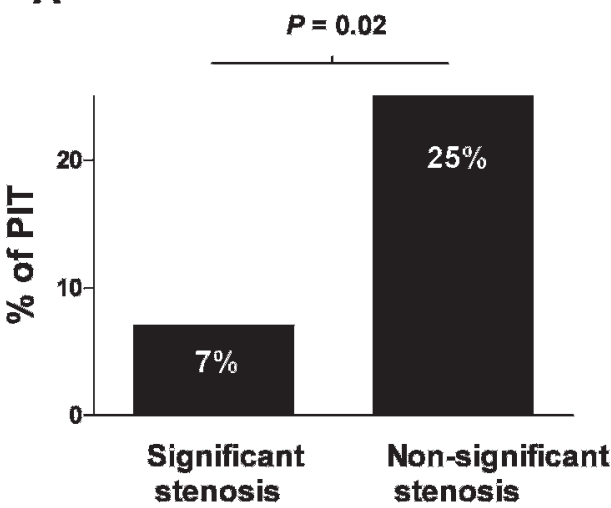

C

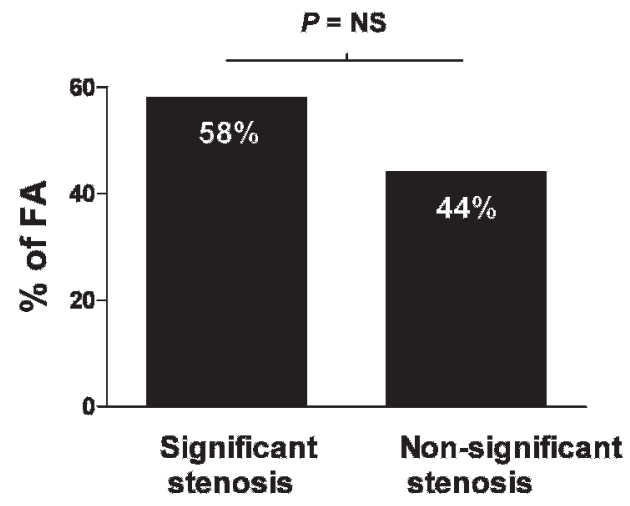

B

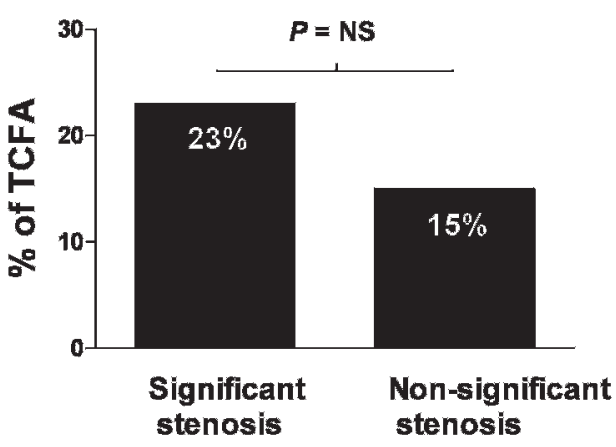

D

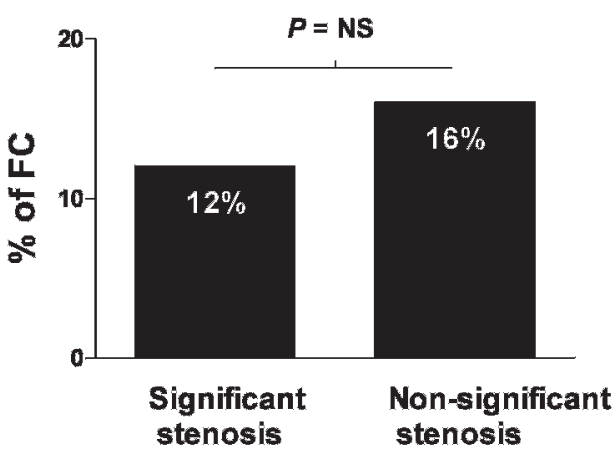


magnetic resonance plaque characterisation and demonstrated that vulnerable lesions were equally present in lesions with significant and non-significant stenosis. ${ }^{28}$

\section{Clinical implications}

At present, MSCT is increasingly used in the evaluation of patients presenting with suspected $\mathrm{CAD}$. Accordingly, the main objective in these patients is to determine the presence of significant stenosis in the coronary arteries and high diagnostic accuracies have been reported for this purpose. ${ }^{29}$ In addition, data supporting its prognostic value are starting to emerge. ${ }^{30} 31$ In several studies the presence of significant CAD on MSCT has been shown to result in a higher likelihood of coronary events. However, assessment of the presence, extent and type of atherosclerosis in addition to the degree of stenosis may potentially further refine risk stratification. In a preliminary prognostic study by Pundziute et al, the presence of nonsignificant stenosis was shown to be associated with worse outcome compared to complete absence of any atherosclerosis. ${ }^{32}$ Our current observations further support this concept, showing no difference in plaque composition and vulnerability between significant and non-significant lesions. Moreover, the incremental prognostic value of MSCT variables describing extent as well as type of atherosclerosis was recently evaluated by van Werkhoven et al. ${ }^{31}$ Interestingly, the authors showed that the presence of a substantial non-calcified plaque burden (regardless of stenosis severity) on MSCT was an independent predictor of adverse cardiac events providing incremental prognostic value over the presence of a significant stenosis. ${ }^{31}$ Accordingly, these as well as our current observations may be of help in understanding the potential value of assessing plaque burden and plaque type on MSCT and suggest that evaluation of MSCT angiograms should not be restricted to assessment of luminal narrowing alone. Possibly, incorporation of information on the presence, extent and composition of atherosclerosis may allow refined and more individualised risk stratification and thus improved identification of patients requiring more aggressive therapy. However, available data are scarce and need to be confirmed in larger patient cohorts.

\section{Limitations}

Imaging of severely stenotic or occluded lesions with IVUS is not possible. Therefore, the present study may not reflect the true spectrum of lesions with significant stenosis.

\section{CONCLUSION}

No evident relation between the degree of stenosis and plaque composition or vulnerability, as evaluated non-invasively by MSCT and invasively by VH IVUS, was observed. Evaluation of plaque composition may provide valuable information incremental to assessment of the degree of stenosis.

Funding: JEvV is supported by the Netherlands Heart Foundation (The Hague, The Netherlands), grant no 2007B223. FRdG is co-supported by the Dutch Technology Foundation STW (Utrecht, The Netherlands), Applied Science Division of NWO and the Technology Program of the Ministry of Economic Affairs, grant no 10084. GP has a training fellowship grant from European Society of Cardiology. MJS has research grants from Boston Scientific (Natick, USA), Medtronic (Minneapolis, United States) and Biotronik (Berlin, Germany). JJB has research grants from Medtronic (Minneapolis, United States), Boston Scientific (Natick, United States), BMS medical imaging (New York, United States), St Jude Medical (St Paul, United States), GE Healthcare (Chalfont St Giles, United Kingdom), Biotronik (Berlin, Germany), and Edwards Lifesciences (Irvine, United States)

Competing interests: None declared.

Provenance and peer review: Not commissioned; externally peer reviewed.

\section{REFERENCES}

1. Manoharan G, Ntalianis A, Muller 0 , et al. Severity of coronary arterial stenoses responsible for acute coronary syndromes. Am J Cardiol 2009;103:1183-8.

2. Shah PK. Mechanisms of plaque vulnerability and rupture. J Am Coll Cardiol 2003:41:15-22S.

3. Ambrose JA, Tannenbaum MA, Alexopoulos D, et al. Angiographic progression of coronary artery disease and the development of myocardial infarction. J Am Coll Cardiol 1988;12:56-62.

4. White CW, Wright CB, Doty DB, et al. Does visual interpretation of the coronary arteriogram predict the physiologic importance of a coronary stenosis? N Eng/ J Med 1984;310:819-24.

5. Virmani R, Burke AP, Kolodgie FD, et al. Pathology of the vulnerable plaque. J Am Coll Cardiol 2006;47:C13-8.

6. Kullo IJ, Edwards WD, Schwartz RS. Vulnerable plaque: pathobiology and clinical implications. Ann Intern Med 1998;129:1050-60.

7. Leber AW, Knez A, Becker A, et al. Accuracy of multidetector spiral computed tomography in identifying and differentiating the composition of coronary atherosclerotic plaques: a comparative study with intracoronary ultrasound. J Am Coll Cardiol 2004;43:1241-7.

8. Schroeder S, Kopp AF, Baumbach A, et al. Noninvasive detection and evaluation of atherosclerotic coronary plaques with multislice computed tomography. J Am Coll Cardiol 2001;37:1430-5

9. Henneman MM, Schuijf JD, Pundziute G, et al. Noninvasive evaluation with multislice computed tomography in suspected acute coronary syndrome: plaque morphology on multislice computed tomography versus coronary calcium score. J Am Coll Cardiol 2008;52:216-22.

10. Pundziute G, Schuijf JD, Jukema JW, et al. Evaluation of plaque characteristics in acute coronary syndromes: non-invasive assessment with multi-slice computed tomography and invasive evaluation with intravascular ultrasound radiofrequency data analysis. Eur Heart J 2008;29:2373-81.

11. Nair A, Kuban DB, Tuzcu EM, et al. Coronary plaque classification with intravascular ultrasound radiofrequency data analysis. Circulation 2002;106:2200-6.

12. Rodriguez-Granillo GA, Garcia-Garcia HM, Mc Fadden EP, et al. In vivo intravascular ultrasound-derived thin-cap fibroatheroma detection using ultrasound radiofrequency data analysis. J Am Coll Cardiol 2005;46:2038-42.

13. Executive Summary of The Third Report of The National Cholesterol Education Program (NCEP) Expert Panel on Detection, Evaluation, And Treatment of High Blood Cholesterol In Adults (Adult Treatment Panel III). JAMA 2001;285:2486-97.

14. Schuijf JD, Pundziute G, Jukema JW, et al. Diagnostic accuracy of 64-slice multislice computed tomography in the noninvasive evaluation of significant coronary artery disease. Am J Cardiol 2006;98:145-8.

15. Austen WG, Edwards JE, Frye RL, et al. A reporting system on patients evaluated for coronary artery disease. Report of the Ad Hoc Committee for Grading of Coronary Artery Disease, Council on Cardiovascular Surgery, American Heart Association. Circulation 1975:51:5-40.

16. Nasu K, Tsuchikane E, Katoh 0, et al. Accuracy of in vivo coronary plaque morphology assessment: a validation study of in vivo virtual histology compared with in vitro histopathology. J Am Coll Cardiol 2006;47:2405-12.

17. Mintz GS, Nissen SE, Anderson WD, et al. American College of Cardiology Clinical Expert Consensus Document on Standards for Acquisition, Measurement and Reporting of Intravascular Ultrasound Studies (IVUS). A report of the American College of Cardiology Task Force on Clinical Expert Consensus Documents. J Am Coll Cardiol 2001:37:1478-92.

18. Carlier SG, Mintz GS, Stone GW. Imaging of atherosclerotic plaque using radiofrequency ultrasound signal processing. J Nucl Cardiol 2006;13:831-40.

19. Virmani R, Kolodgie FD, Burke AP, et al. Lessons from sudden coronary death: a comprehensive morphological classification scheme for atherosclerotic lesions. Arterioscler Thromb Vasc Biol 2000;20:1262-75.

20. Hodgson JM, Reddy KG, Suneja R, et al. Intracoronary ultrasound imaging: correlation of plaque morphology with angiography, clinical syndrome and procedural results in patients undergoing coronary angioplasty. J Am Coll Cardiol 1993;21:35-44.

21. O'Rourke RA, Brundage BH, Froelicher VF, et al. American College of Cardiology/ American Heart Association Expert Consensus document on electron-beam computed tomography for the diagnosis and prognosis of coronary artery disease. Circulation 2000;102:126-40

22. Sipahi I, Tuzcu EM, Moon KW, et al. Do the extent and direction of arterial remodelling predict subsequent progression of coronary atherosclerosis? A serial intravascular ultrasound study. Heart 2008;94:623-7.

23. Mintz GS, Pichard AD, Popma JJ, et al. Determinants and correlates of target lesion calcium in coronary artery disease: a clinical, angiographic and intravascular ultrasound study. J Am Coll Cardiol 1997;29:268-74.

24. Motoyama S, Kondo T, Sarai M, et al. Multislice computed tomographic characteristics of coronary lesions in acute coronary syndromes. J Am Coll Cardiol 2007:50:319-26.

25. Schuijf JD, van Werkhoven JM, Pundziute G, et al. Invasive versus noninvasive evaluation of coronary artery disease. JACC Cardiovasc Imaging 2008;1:190-9.

26. Giroud D, Li JM, Urban P, et al. Relation of the site of acute myocardial infarction to the most severe coronary arterial stenosis at prior angiography. Am J Cardiol 1992;69:729-32.

27. Yamagishi M, Terashima M, Awano K, et al. Morphology of vulnerable coronary plaque: insights from follow-up of patients examined by intravascular ultrasound before an acute coronary syndrome. J Am Coll Cardiol 2000;35:106-11. 
28. Saam T, Underhill HR, Chu B, et al. Prevalence of American Heart Association type VI carotid atherosclerotic lesions identified by magnetic resonance imaging for different levels of stenosis as measured by duplex ultrasound. J Am Coll Cardiol 2008;51:1014-21.

29. Budoff MJ, Dowe D, Jollis JG, et al. Diagnostic performance of 64-multidetector row coronary computed tomographic angiography for evaluation of coronary artery stenosis in individuals without known coronary artery disease: results from the prospective multicenter ACCURACY (Assessment by Coronary Computed

Tomographic Angiography of Individuals Undergoing Invasive Coronary Angiography) trial. J Am Coll Cardiol 2008;52:1724-32.
30. Min JK, Shaw LJ, Devereux RB, et al. Prognostic value of multidetector coronary computed tomographic angiography for prediction of all-cause mortality. J Am Coll Cardiol 2007; 50:1161-70.

31. Van Werkhoven JM, Schuijf JD, Gaemperli 0, et al. Prognostic value of multislice computed tomography and gated single-photon emission computed tomography in patients with suspected coronary artery disease. J Am Coll Cardiol 2009;53:623-32.

32. Pundziute G, Schuijf JD, Jukema JW, et al. Prognostic value of multislice computed tomography coronary angiography in patients with known or suspected coronary artery disease. J Am Coll Cardiol 2007;49:62-70. 